\title{
Sharing Knowledge about Open Source Licenses at DLR
}

\author{
Andreas Schreiber \\ German Aerospace Center \\ (DLR) \\ Cologne, Germany \\ andreas.schreiber@dlr.de

\section{Carina Haupt} \\ German Aerospace Center \\ (DLR) \\ Berlin, Germany \\ carina.haupt@dlr.de
}

Permission to make digital or hard copies of part or all of this work for personal or

\begin{abstract}
In science, more and more software is published as Open Source software or uses Open Source projects. Therefore, scientists must be aware of requirements, restrictions and compatibility issues of Open Source licenses. We describe our strategy at the German Aerospace Center (DLR) to awake the awareness among our domain scientists and to enable and support them in publishing Open Source software, which is free of license issues. We provide hands-on material, offer training courses, and foster knowledge sharing between peer scientists. Findings and feedback prove the usefulness and effectiveness.
\end{abstract}

\section{ACM Classification Keywords}

K.5.1. [Legal Aspects of Computing]: Licensing; K.6.1.

[Management of Computing and Information Systems]: Training

\section{Author Keywords}

open source;licensing;collaboration

\section{Introduction}

The German Aerospace Center ( $D L R)$ is a large governmental research organization with approximately 8000 employees at 20 sites. Between 2000 and 3000 persons are developing software in part of their work [5]. Most of them do not have any specific education in software 


\section{Examples of typical DLR Open Source projects}

\section{ESMVALTOOL (The Earth} System Model eValuation Tool) [2] is a data science tool for the evaluation of Earth System Models that allows for routine comparison of single or multiple models, either against predecessor versions or against observations. It is developed since 2008 and has grown to a community tool with 20 participating organization.

SUMO (Simulation of Urban MObility) [3] is a simulation tool for microscopic,

space-continuous road traffic simulations. Development started around 1998 and it is now used by many research groups who are contributing extensions.

RepoGuard (Repository Guard) [4] is an example for a small software engineering tool. RepoGuard performs checks and other activities triggered by check-ins in version management systems. development but only some programming skills. Especially, their knowledge about Open Source and adjacent topics is very limited.

The lack of knowledge about licensing [1] led to many issues because employees and management were confronted with making decisions anyway (e.g., choosing a proper license). Often, software was not published due to fear of license violations or existing Open Source software was not used because of potential negative consequences.

We support domain scientists at DLR by enabling them to obtain the needed information themselves and make well-founded decisions with the following:

- Information resources and training courses about Open Source licensing.

- Collaboration tools (Wikis) and dedicated workshops for knowledge sharing among peer scientists.

\section{Information Resources and Education}

A major reason among scientists at DLR for problems and issues in past was lack of knowledge about Open Source licensing. To overcome this situation, we provided a printed brochure and regular training courses about legal aspects of Open Source.

\section{Open Source Brochure}

We designed the DLR Open Source brochure as a reference book for all employees. An external law firm developed it in cooperation with DLR. The brochure was released in 2013 and distributed as printed copies and download-able PDF documents.

The brochure helps DLR employees to answer the most basic questions related to Open Source Software licenses. Specifically, it describes how to proceed when Open Source software should be distributed to third-parties. For the distribution of unmodified and modified Open Source Software, the brochure lists all obligations of the most important licenses that are used at DLR. The licenses are grouped into licenses with strong copyleft, weak copyleft, without copyleft, and with special rights. For each license, the brochure has a simple checklist with all required obligations (e.g., how to prepare source code or which information to add to the distribution) as well as text boxes with explanations and additional information.

\section{Trainings}

Regular trainings are part of the education program of DLR since 2012. The trainings take place in different DLR locations across Germany (see Table 1). Several additional customized trainings took place for specific teams.

The curriculum of the training consists of several topics about Open Source with focus on legal concepts and issues, such as copyright and liability, as well as Open Source licenses, including a detailed discussion of some widely used licenses.

For each training, participants can hand in their expectations and wishes for additional topics before the training and give detailed anonymous feedback after the training. Both on a voluntary basis by using paper questionnaires.

The questionnaire asks for prior knowledge about legal aspects of participants before the training. Half of the participants (50\%) had some very limited knowledge, around a quarter has no knowledge, and another quarter has some knowledge based on previous experiences. The ratings after the training are mostly at least good on average. The results (Figure 1) shows that more than three-quarters of all participants rated the fulfillment of their 


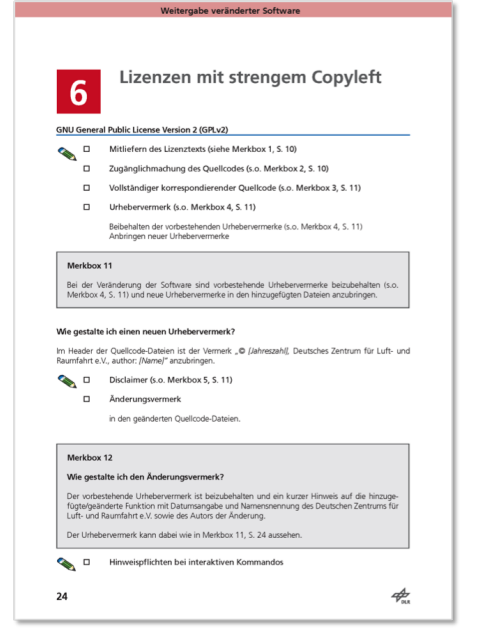

Figure 2: Example page of the

DLR Open Source Brochure.

\begin{tabular}{ccc}
\hline Year & Participants & DLR site \\
\hline 2012 & 32 & Oberpfaffenhofen \\
2013 & 23 & Berlin \& Cologne \\
2014 & 12 & Stuttgart \\
2015 & 9 & Berlin \\
2016 & 14 & Cologne \\
\hline
\end{tabular}

Table 1: Numbers of Open Source training participants. expectations with either "very good" (34.8\%) or "good" (43.6\%). The participants also rated the expected benefits for their daily work. A third rated the expected benefit with "very good" (34.8\%) and almost a half with "good" (43.5\%).

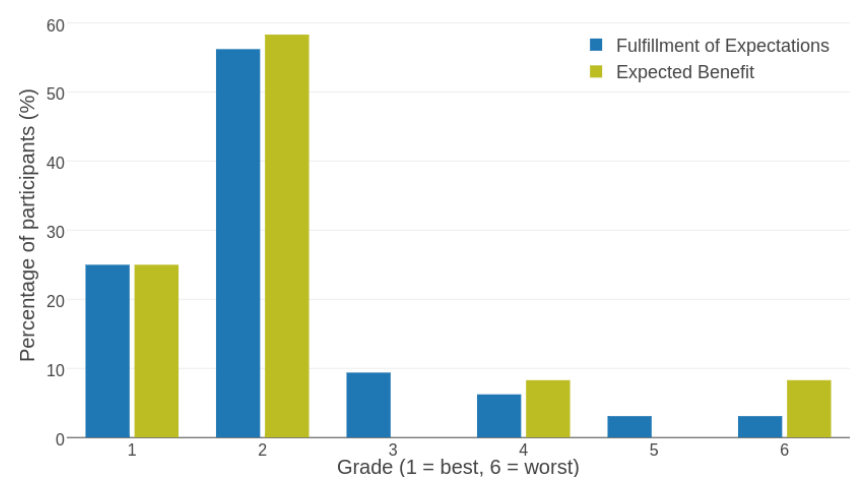

Figure 1: Ratings of Open Source trainings.

\section{Knowledge Sharing}

An important foundation of DLRs Open Source strategy is an environment for sharing knowledge between peer scientists. To foster knowledge sharing, we introduced a Web-based collaboration tool using a Wiki and a workshop series for face-to-face knowledge exchange and discussions.

\section{Wikis}

In 2013, a central Wiki installation was introduced for DLR, which became the focal point for the DLR Open Source community. It provides a single point of access for information and allows anybody of DLR to share their knowledge and experiences.

The information about Open Source is in the DLR.OPEN
Wiki section, which also covers any other "Open" topic such as Open Data, Open Standards, Open Access, or Open Science. The DLR.OpEN Wiki provides pages for topics, tools, literature, questions, and events as well as a separate section about how to get involved. Questions and their answers often are the basis for new pages on topics or tools. Since asking a question is a much lower hurdle to take than starting a page, this section became the entry point for many contributers. The Wiki offers a blog feature to announce new content in the Wiki and to share news (e.g., relevant Call for Papers or press releases).

\section{Knowledge Exchange Workshops}

Since 2013, so called knowledge exchange workshops (german: WissensAustauschWorkshops (WAW)) are held at DLR. These workshops are interdisciplinary and aim on long-term exchange of knowledge on overlapping topics for the establishment and expansion of professional networks.

In 2014, the knowledge exchange workshop series for Software Engineering was started. In 2016, the focus of this workshop series was Open Source. In the same year the workshop series DLR.OPEN started, which aims to cover all "Open" movements. The first workshop focused on Open Source and Open Standards.

In the knowledge exchange workshops, next to learning about Open Source topics, the exchange between DLR employees was encouraged. The peer-to-peer communication was emphasized to extend the informal networking among employees. In session with the whole plenum as well in small groups, the workshops produced many results and finding about the employees experiences, hopes, and fears.

We learned that Open Source software is already widely used at DLR. But mainly as more general tools and 
libraries, which have been included into internal development processes and software projects. Research topic specific software was mainly developed in-house. On the other hand, exactly this specialized tools were seen as candidates to be published under an Open Source license.

As a major result of all workshops, the participants identified the lack of an official DLR process on how to publish Open Source software. One of the action items of the workshops therefore was to start the development of such a process.

\section{Conclusion}

We presented some of our strategical efforts to support domain scientists at DLR in using and publishing Open Source Software. Focus of our activities is to provide usable material and support for immediate actions. We started with an Open Source brochure covering licensing, accompanied by training courses in the regular education program. After that we introduced collaboration platforms for knowledge sharing among peer scientists, such as Wiki pages and knowledge exchange workshops. Since we introduced our Open Source strategy, serious failures and issues with licensing have been reduced note-worthily. Feedback of domain scientist is good or very good. Based on that feedback we will extend our effort in the future, for example, by providing more technical infrastructure for code hosting or automated license checking.

\section{REFERENCES}

1. D. A. Almeida, G. C. Murphy, G. Wilson, and M. Hoye. 2017. Do Software Developers Understand Open Source Licenses?. In 2017 IEEE/ACM 25th International Conference on Program Comprehension (ICPC). 1-11. DOI :

http://dx.doi.org/10.1109/ICPC.2017.7
2. V. Eyring, M. Righi, A. Lauer, M. Evaldsson, S. Wenzel, C. Jones, A. Anav, O. Andrews, I. Cionni, E. L. Davin,

C. Deser, C. Ehbrecht, P. Friedlingstein, P. Gleckler, K.-D. Gottschaldt, S. Hagemann, M. Juckes, S. Kindermann, J. Krasting, D. Kunert, R. Levine, A. Loew, J. Mäkelä, G. Martin, E. Mason, A. S. Phillips, S. Read, C. Rio, R. Roehrig, D. Senftleben, A. Sterl, L. H. van Ulft, J. Walton, S. Wang, and K. D. Williams. 2016. ESMValTool - a community diagnostic and performance metrics tool for routine evaluation of Earth system models in CMIP. Geoscientific Model Development 9, 5 (2016), 1747-1802. DOI : http://dx.doi.org/10.5194/gmd-9-1747-2016

3. D. Krajzewicz, J. Erdmann, M. Behrisch, and L. Bieker. 2012. Recent Development and Applications of SUMO - Simulation of Urban MObility. International Journal On Advances in Systems and Measurements 5, 3\&4 (December 2012), 128-138.

4. M. Legenhausen, S. Pielicke, J. Rühmkorf, H. Wendel, and A. Schreiber. 2009. RepoGuard: A Framework for Integration of Development Tools with Source Code Repositories.. In 2009 Fourth IEEE International Conference on Global Software Engineering. IEEE Computer Society, 328-331. DOI : http://dx.doi.org/10.1109/ICGSE. 2009.51

5. A. Schreiber, R. Galoppini, M. Meinel, and T. Schlauch. 2014. An Open Source Software Directory for Aeronautics and Space. In Proceedings of The International Symposium on Open Collaboration (OpenSym '14). ACM, New York, NY, USA, Article 46, 7 pages. DOI :

http://dx.doi.org/10.1145/2641580.2641630 